\title{
A Randomized Trial of Combined PD-L1 and CTLA-4 Inhibition with Targeted Low-Dose or Hypofractionated Radiation for Patients with Metastatic Colorectal Cancer
}

\author{
Arta M. Monjazeb ${ }^{1}$, Anita Giobbie-Hurder ${ }^{2}$, Ana Lako ${ }^{3}$, Emily M. Thrash ${ }^{2}$, Ryan C. \\ Brennick $^{2}$, Katrina Z. Kao ${ }^{2}$, Claire Manuszak ${ }^{2}$, Ryan D. Gentzler ${ }^{4}$, Anteneh Tesfaye ${ }^{5}$, Salma \\ K. Jabbour ${ }^{6}$, Olatunji B. Alese ${ }^{7}$, Osama E. Rahma ${ }^{2,3}$, James M. Cleary ${ }^{2,3}$, Elad Sharon ${ }^{8}$, \\ Harvey J. Mamon ${ }^{2,3}$, May Cho ${ }^{1}$, Howard Streicher ${ }^{8}$, Helen X. Chen ${ }^{8}$, Mansoor M. Ahmed ${ }^{9}$, \\ Adrian Mariño-Enríquez ${ }^{2,3}$, Seunghee Kim-Schulze ${ }^{10}$, Sacha Gnjatic ${ }^{10}$, Emanual \\ Maverakis $^{11}$, Alina I. Marusina ${ }^{11}$, Alexander A. Merleev ${ }^{11}$, Mariano Severgnini ${ }^{2}$, Kathleen L. \\ Pfaff $^{2}$, James Lindsay ${ }^{2}$, Jason L. Weirather ${ }^{2}$, Srinika Ranasinghe ${ }^{2}$, Alexander Spektor ${ }^{2,3}$, \\ Scott J. Rodig ${ }^{2,3}$, Stephen F. Hodi ${ }^{2,3}$, Jonathan D. Schoenfeld ${ }^{2,3}$ \\ ${ }^{1}$ Department of Radiation Oncology, University of California Davis, Comprehensive Cancer \\ Center, Sacramento, CA \\ ${ }^{2}$ Dana-Farber Cancer Institute, Boston, MA \\ ${ }^{3}$ Brigham and Women's Hospital, Boston, MA \\ ${ }^{4}$ University of Virginia, Charlottesville, VA \\ ${ }^{5}$ Karmanos Cancer Institute/Wayne State University, Detroit, MI \\ ${ }^{6}$ Rutgers Robert Wood Johnson Medical School, New Brunswick, NJ \\ ${ }^{7}$ Winship Cancer Institute of Emory University, Atlanta, GA \\ ${ }^{8}$ Cancer Therapy Evaluation Program, National Cancer Institute, Bethesda, MD \\ ${ }^{9}$ Radiation Research Program, National Cancer Institute, Bethesda, MD \\ ${ }^{10}$ Mount Sinai School of Medicine, New York, NY \\ ${ }^{11}$ Department of Dermatology, University of California Davis, School of Medicine, Sacramento, CA
}

\section{Abstract}

Purpose: Prospective human data are lacking regarding safety, efficacy and immunologic impacts of different radiation doses administered with combined PD-L1/CTLA-4 blockade.

Experimental Design: We performed a multicenter phase 2 study randomizing subjects with metastatic microsatellite-stable colorectal cancer (CRC) to repeated low-dose fractionated RT (LDFRT) or hypofractionated radiation (HFRT) with PD-L1/CTLA-4 inhibition. The primary endpoint was response outside the radiation field. Correlative samples were analyzed using 
multiplex immunofluorescence (IF), immunohistochemistry, RNA/ T-cell receptor (TCR) sequencing, CyTOF, and Olink.

Results: Eighteen patients were evaluable for response. Median lines of prior therapy were 4 (range 1-7). Sixteen patients demonstrated toxicity potentially related to treatment (84\%), and 8 patients had grade 3-4 toxicity (42\%). Best response was stable disease in one patient with out-offield tumor shrinkage. Median OS was 3.8 months (90\% CI:2.3-5.7 months). Correlative IF and RNAseq revealed increased infiltration of CD8+, and CD8+/PD1+/Ki67+ T-cells in the radiation field after HFRT. LDFRT increased foci of micronuclei / primary nuclear rupture in 2 subjects. CyTOF and RNAseq demonstrated significant declines in multiple circulating immune populations particularly in patients receiving HFRT. TCR-sequencing revealed treatment associated changes in T-cell repertoire in the tumor and peripheral blood.

Conclusion: We demonstrate the feasibility and safety of adding LDFRT and HFRT to PD-L1/ CTLA-4 blockade. Although the best response of stable disease doesn't support the use of concurrent PD-L1/CTLA-4 inhibition with HFRT or LDFRT in this population, biomarkers provide support that both LDFRT and HFRT impact the local immune microenvironment and systemic immunogenicity that can help guide future studies.

\section{Introduction}

Although immune checkpoint inhibition has led to significant clinical benefit in patients with microsatellite instable (MSI-H) colorectal cancer, $(1,2)$ the majority of patients with microsatellite stable disease (MSS) have proven largely refractory to single agent PD-1 blockade and other immunotherapies including combined PD-1/CTLA-4 blockade.(1,2) Thus, strategies are needed to help engender antitumor immunity and increase response rates. Focal radiation demonstrates immune stimulating effects in animal models and anecdotal clinical reports,(3) including the potential ability to increase response to combined PD(L)-1 and CTLA-4 blockade.(4)

We performed a randomized multi-center phase 2 study with the goal of evaluating the safety and efficacy of two different radiation regimens given in combination with combined PD-L1 / CTLA-4 blockade in patients with MSS colorectal cancer who had progressed on at least one line of prior systemic chemotherapy. A hypofractionated radiation regimen of 24 Gy given over 3 fractions (HFRT) was adapted from preclinical studies demonstrating positive immune effects in combination with immune checkpoint blockade,(5) including in tumor types that were otherwise unresponsive to immune checkpoint therapy.(4) A lower dose hyperfractionated arm of $0.5 \mathrm{~Gy}$ was administered twice daily for 2 days and then repeated during each of the first 4 cycles of therapy (LDFRT) to investigate immune effects of a regimen similar to that used in preclinical models(6) and pilot clinical studies, $(7,8)$ but unlikely to have pronounced cytotoxic effects directly or lead to significant toxicity. Correlative studies were performed on circulating blood and tumor biopsies to examine the immunologic impacts of these different regimens. 


\section{Materials and Methods}

\section{Participants and eligibility}

Patients with histologically confirmed metastatic microsatellite stable colorectal adenocarcinoma who had progressed on at least one line of chemotherapy were recruited to 6 centers in the NCI Experimental Therapeutics Clinical Trials Network (ETCTN) to ETCTN protocol 10021 (Clinicaltrials.gov identifier NCT02888743). Investigators obtained written informed consent from each participant prior to enrollment. The research was conducted in accordance with the recognized ethical guidelines of the Declaration of Helsinki, CIOMS, Belmont Report, and U.S. Common Rule. Clinical study was performed after approval by a central institutional review board. Patients were aged $\geq 18$ years and had adequate hepatic, bone marrow, and renal organ function and an Eastern Cooperative Oncology Group (ECOG) performance status of 0 or 1 . All patients had measurable disease including one liver lesion that could be targeted by radiation in the context of this trial and at least one other site of disease outside of the radiotherapy field for response assessment. To be eligible, the liver lesion to be irradiated within the context of this trial could not have been the target of previous radiation therapy. Subjects also needed at least one additional lesion outside of the radiation treatment field (more than one lesion permitted) that could be subsequently monitored for systemic (out-of-field) treatment response. Patients were confirmed to have microsatellite stable disease documented by either immunohistochemistry that did not suggest loss of MLH-1, MSH-2, PMS2 or MSH6 or PCR testing that did not suggest microsatellite instability.

\section{Procedures and screening}

Patients were required to either undergo a fresh tumor biopsy for the purposes of screening or provided an archival tumor sample obtained less than 3 months prior to study enrollment. Baseline screening included a CT of the chest abdomen and pelvis for staging. All patients underwent CT-based planning for radiation of 1-2 lesions within the liver with intravenous contrast and internal tumor and organ motion management. 4D-CT planning and image guided radiation therapy was mandatory for patients randomized to HFRT. The use of 4DCT and IGRT was not mandated for subjects receiving low-dose radiation therapy. Radiation was administered with an optional $0-1 \mathrm{~cm}$ clinical tumor volume margin to account for any uncertainty or microscopic disease and all patients were treated with a $5 \mathrm{~mm}$ planning tumor volume.

\section{Study design and treatment}

The primary objective of the trial was to determine the overall response rate in the two treatment arms according to RECIST v1.1 criteria (9) and excluding the lesions that had been irradiated. Patients were randomized 1:1 upon study entry to a treatment regimen consisting of the PD-L1 inhibitor durvalumab administered at a fixed dose of $1500 \mathrm{mg}$ every 4 weeks for a maximum of 13 cycles and the CTLA- 4 inhibitor tremelimumab administered at a fixed dose of $75 \mathrm{mg}$ every 4 weeks for a maximum of 4 cycles combined with either low-dose fractionated radiation therapy (LDFRT) or hypofractionated radiation therapy (HFRT). The treatment regimens are shown in Supplemental Figure 1. LDFRT consisted of a dose of 2 Gy administered in 4 fractions over 2 days repeated for the first 4 cycles of therapy 
(total dose 8 Gy). HFRT consisted of a total dose of 24 Gy administered in three 8 Gy fractions no more frequently than every other day during the first cycle of therapy only. Radiation was administered the week following durvalumab/tremelimumab. Patients were evaluated for response every 12-weeks after an initial restaging scan at 7-8 weeks. Best overall response was best RECIST response observed between the start of treatment until disease progression or recurrence.

Secondary endpoints included progression-free survival and overall survival. Overall survival was the time interval between study enrollment and death from any cause. For patients lost to follow-up or who had no documentation of death at the time of analysis, follow-up was censored at the date of last assessment of vital status. Progression-free survival was the time from enrollment to the earlier of objective disease progression or death. For patients without progression, follow-up was censored at the date of last adequate restaging, unless death occurred within 12 weeks following the date last known progressionfree, in which case the death was counted as a PFS event.

Correlative blood samples were obtained prior to all study treatment and then again prior to cycle 2 of durvalumab/tremelimumab. On- treatment biopsies of irradiated lesions were obtained during week 7 or 8 (between cycles 2 and 3) and around the time of the first restaging scans (Supplemental Figure 1). Toxicities were graded according to CTCAE v4.0.

\section{Statistical design}

This phase 2 study was designed using two parallel, Simon optimal two-stage designs to identify combinations capable of effecting a systemic response outside of the radiation treatment field according to RECIST v1.1 criteria. The goal was to identify a promising rate of response of $20 \%$ (null rate 5\%), with a type-I error of 5\% and $80 \%$ power. Enrollment was stopped after 10 patients were enrolled in each arm in the first stage of the study according to prespecified stopping criteria looking for at least 1 objective response before proceeding to the second stage.

The distributions of overall and progression-free survival were summarized using the method of Kaplan-Meier and compared using log-rank tests.

\section{Correlative studies}

PD-L1 immunohistochemistry, multiplex immunofluorescence (mIF), cytometry by time of flight (CyTOF) and Olink correlative studies were performed through the Cancer Immune Monitoring and Analysis Centers (CIMAC) Immuno-Oncology Biomarkers Network using analytically-validated and standardized platforms. Detailed standard operating procedures are available at https://cimac-network.org/assays/ and additional detail is provided in the Supplemental Methods and Supplementary Table 6. PD-L1 immunohistochemistry was performed using the 9A11 antibody clone (Cell Signaling Technologies, Danvers MA) using the BOND Max by Leica Biosystems. PD-L1 H-score was determined by multiplying the percentage of tumor cells staining positive for PD-L1 by the intensity as determined on a 1+, $2+, 3+$ scale. Multiplex immunofluorescence used formalin fixed paraffin embedded slides 
of the primary tumor stained using BOND RX automated stainer using published protocols. (11-13).

Peripheral blood mononuclear cells (PBMCs) were analyzed using CyTOF with reference sample spike-in and palladium-based mass tag cell barcoding of individual samples as previously described.(14,15) Serum cytokines were analyzed using Olink multiplex assay platform with Immuno-oncology panel (Olink Bioscience, Uppsala, Sweden), according to the manufacturer's instructions. The inflammatory panel includes 92 proteins associated with immune response. Additional detail is provided in the supplementary methods. Analyses of changes in circulating biomarkers over time were based on longitudinal mixed models with intervention arm, time and the interaction of intervention and time as independent predictors. Biomarker studies were exploratory; there were no adjustments made for multiple comparisons.

RNA sequencing was performed as previously described and techniques and analyses are described in more detail in the supplementary methods.(16) Differential expression p-values were corrected for multiple testing using the false discovery rate (FDR) method. Genes with FDR-adjusted $\mathrm{p}$ values $<0.05$, and fold change $>2$ or $<0.5$ were considered differentially expressed. To calculate the number of expanded/contracted clones we performed statistical analysis of each TCR clones abundance for each patients before and after treatment using "fisher.test" $\mathrm{R}$ function. Clones which showed p-value less than 0.05 in the test were counted as contracted or expanded.

\section{Results}

\section{Treatment with combined PD-L1/CTLA-4 and low- or hypofractionated radiation was tolerated but did not induce systemic responses}

Twenty subjects were enrolled across 6 centers in the United States from August to November 2017, with 10 subjects randomized to each arm (Supplementary Figure 2).

One patient subsequently withdrew before starting therapy and was excluded from all subsequent analyses. Baseline demographics are summarized in Supplemental Table 1. Subjects were well balanced with regards to gender and age. All patients had previously been treated with surgery and 5 had received prior radiation therapy locations that were not subsequently irradiated on this trial. The median number of prior lines of therapy was 4 (range 1-7). Sixteen patients had toxicity at least possibly related to therapy; there were 6 patients with grade 3 toxicities and 3 with grade 4 (Supplemental Table 2). The overall rate of Grade 3-4 adverse events that were deemed at least possibly related to therapy was 0.42 (90\% CI: 0.23 to 0.63$)$; these occurred in 3 of 10 patients who received HFRT $(0.30,90 \%$ exact CI 0.09-0.61), and 5 of 9 patients treated with LDFRT $(0.56,90 \%$ CI $0.25-0.83)$.

No objective responses outside of the radiation field were observed. Two patients were unevaluable for response ( 1 patient withdrew before treatment as above and another patient withdrew after treatment but before restaging with disease related toxicity) and 1 patient demonstrated stable disease. The subject with stable disease had previously progressed on 6 prior lines of therapy and demonstrated a response in an unirradiated porta hepatis lymph 
node following HFRT and durvalumab/tremelimumab in the setting of decreasing overall tumor burden before being removed from study treatment after 4 cycles because of symptomatic new lesions (Supplemental Figure 3). Median follow-up was 3.9 months. Median overall survival was 3.8 months (90\% CI: 2.3-5.7 months) and progression-free survival was 1.7 months (90\% CI: 1.5-1.8 months), with no significant differences between arms (Supplemental Figure 4).

\section{Immunohistochemistry and multiplex immunofluorescence revealed changes in T-cell and macrophage infiltration that were radiation dose dependent and micronuclei / primary nuclear rupture formation}

Sixteen patients had baseline specimens for PD-L1 assessment. Baseline expression of PDL1 was relatively minimal (tumor proportion score, TPS $<=5$; H-score $<=10$ out of a maximum possible score of 300) in all patients. A TPS score of 5 / H-score of 10 was seen in 2 subjects, including the subject who demonstrated an out-of-field response on trial. Additionally, comparing matched baseline and on treatment specimens, the change in PD-L1 expression was minimal $(<2)$ or none among all 5 subjects with evaluable pre and ontreatment samples.

Multiplex immunofluorescence (IF) evaluated levels of CD4, CD8, PD-1 and Ki67 expressing cell populations (Figure 1). Eighteen patients had tissue for baseline evaluation. Baseline levels were highly variable (CD8+ T-cell range 11-107 cells / mm2, PD-1 cell range 0-209 cells / mm2). The highest levels of tumor-infiltrating CD8+ and PD-1+ cells were observed in the hepatic lesion that was to be irradiated in the patient who subsequently demonstrated an out-of-field tumor response (Figure 1A). There were no significant differences in baseline immune populations analyzed among patients who received prior radiation $(\mathrm{n}=5)$ and others $(\mathrm{n}=13)$, with a non-significant difference in baseline infiltration of CD4+ and CD8+ T-cells (median CD8+/CD4+ T-cells: 77 cells / mm2 and 214 cells /mm2 for patients who received prior radiation, and 21 cells $/ \mathrm{mm} 2$ and 1122 cells $/ \mathrm{mm} 2$ for patients who did not receive prior radiation, $\mathrm{p}=0.17$, and $\mathrm{p}=0.20$, respectively). Evaluation of baseline and on-treatment samples in five patients demonstrated that the study treatment led to an increase in CD8+ T-cells ( $\mathrm{p}=0.01$ and $\mathrm{p}=0.06$ ) as well as CD8+/PD1+/Ki67+ T-cells $(\mathrm{p}=0.004$ and $\mathrm{p}=0.004)$ among two patients who received HFRT (Figure 1B, C). There were no significant increases among LDFRT patients $(\mathrm{N}=3)$.

Gene set variation analysis of RNA-Seq data corroborated these findings. Specifically, there were no differences between treatment arms in the expression of T cell, CD8+ T cell, and Th-1 T cell gene sets at baseline but trends towards increases after HFRT in comparison to LFRT were noted (Supplemental Figure 5). Interestingly, this trend was also observed for NK cells (Supplemental Figure 5).

We also interrogated other immune populations and immune effects of radiation on these paired samples obtained from HFRT and LDFRT patients. Although there was interpatient variability, the ratio of M1 / M2 macrophages in the tumor microenvironment decreased in two of two patients receiving HFRT and increased in all three patients treated with LDFRT (Figure 2) suggesting an influence of RT dose / fractionation on macrophage polarization. We also used IF to identify co-localization of cGAS and DAPI outside of the nucleus to 
score micronuclei and cGAS with a corresponding nuclear DAPI defect in the nuclear rim to score primary nuclear ruptures (PNR, Figure 3). These analyses demonstrated that 2 out of 3 patients treated with LDFRT demonstrated increases in micronuclei / PNR (3.7 and 5.5 fold increase, $\mathrm{p}=0.04$ and $\mathrm{p}<0.0001$, respectively), while numbers remained relatively stable or slightly decreased at the 7-8 week timepoint in the other LDFRT and two HDRT patients $(0.76,0.83$ and 0.84 fold change, $\mathrm{p}=0.81, \mathrm{p}=54$ and $\mathrm{p}=0.68$, respectively).

Macrophage markers in the tumor microenvironment were further evaluated in RNA-Seq data from paired tumor biopsy RNA-seq. Gene set variation analysis of data was evaluated for macrophage gene marker sets. In the HFRT cohorts there was a significant increase in macrophage markers post-treatment, but no differences were observed in the LDFRT group (Supplemental Figure 6). We additionally examined macrophage polarization genes ARG1, NOS2, IL10, and CD163. In the HFRT group we also observed a significant decrease in ARG1 post-treatment, but no significant differences were seen in NOS2, IL10, and CD163 or in the LDFRT cohort.

\section{Treatment induced changes in systemic immune markers were also impacted by radiation dose and included declines in effector T-cell populations}

Blood was collected prior to treatment and then in conjunction with cycle 2 in 19 patients. Initial analyses of overall impacts on white blood cell counts (WBC) demonstrated minimal overall changes in both arms (Supplemental Table 3). There were non-significant declines in absolute lymphocytes counts in both arms that were more pronounced following HFRT (Figure 4A). CyTOF was employed to further analyze specific cell populations. Results on matched PBMC samples from 12 patients revealed significant declines in multiple CD4+ and CD8+ cell populations following HFRT compared with LDFRT including global $\mathrm{CD} 3+\mathrm{CD} 4+(\mathrm{p}=0.004), \mathrm{CD} 3+\mathrm{CD} 8+(\mathrm{p}=0.02)$, and CD4+ and CD8+ T-cells expressing markers of activation, including $\mathrm{CD} 8+\mathrm{CXCR} 3+(\mathrm{p}=0.003), \mathrm{CD} 4+\mathrm{ICOS}+(\mathrm{p}=0.02)$, $\mathrm{CD} 4+\mathrm{Lag} 3+(\mathrm{p}=0.03), \mathrm{CD} 4+\mathrm{CXCR} 3+(\mathrm{p}=0.002), \mathrm{CD} 4+41 \mathrm{BB}+(\mathrm{p}=0.003), \mathrm{CD} 4+\mathrm{CRTH} 2+$ $(\mathrm{p}=0.004), \mathrm{CD} 4+\mathrm{GITR}+(\mathrm{p}=0.03), \mathrm{CD} 4+\mathrm{OX} 40+(\mathrm{p}=0.003)$, (Figure 4B,C). In contrast, $\mathrm{T}$ cell populations generally remained stable or increased in patients who received LDFRT compared with HFRT, with increases in CD8+CD40L+ $(\mathrm{p}=0.002)$, and decreases in TIGIT+ T-regulatory cells (defined as CD3+CD19-CD4+CD8-CD127low CD25+) ( $\mathrm{p}=0.0008)$.

We used the Olink multiplex immune-oncology panel to interrogate changes induced by cycle 2 of treatment in 92 measured cytokines and chemokines as compared to pretreatment levels among matched samples from 13 patients. Of these, 34 demonstrated significant increases over the course of treatment in both arms (Supplemental Figure 7). These included known mediators of anti-tumor immunity such as interferon-gamma ( $\mathrm{p}=0.01)$, CCL20 ( $\mathrm{p}=0.002)$, CXCL-9, 10, 11 ( $\mathrm{p}=0.001, \mathrm{p}=0.0001$, and $\mathrm{p}=0.030$, respectively), as well as others such as PD-L1 ( $\mathrm{p}<0.0001$ HDFRT, $\mathrm{p}=0.0006$ LDFRT).

\section{Treatment was associated with differential expression of immune genes in the tumor microenvironment and peripheral blood}

We next further examined associations between study therapy and gene expression, both within circulating PBMCs and in the tumor microenvironment, by performing RNA-Seq of 
pre- and post-therapy samples obtained from 5 patients for whom sufficient tissue was available. When pre to post-treatment samples were compared, there were 407 differentially expressed genes (FDR $\leq 0.05, \mathrm{FC} \geq|2|$ ) in PBMCs (Figure 5, Supplemental Table 4). Therapy induced gene expression differences were also observed in in the tumor microenvironment with 445 differentially expressed genes (Supplemental Figure 8, Supplemental Table 5). Principal component analysis of tumor microenvironment gene expression data largely separated pre- and post-treatment biopsy specimens (Supplemental Figure 8B). Examination of the 4 paired biopsy specimens by cluster analysis of the top 30 most variable expressed genes confirms the results of the PCA analysis, showing most samples clustering by pre- vs. post-treatment (Supplemental Figure 8C). We also examined the consensus molecular subtypes of these paired biopsies pre- and post- treatment (17) to ascertain if therapy impacted the subtype. Three of four samples were CMS4 at baseline, the 4th sample could not be aligned with a subtype at baseline, but was most closely aligned with CMS4. All four samples remained CMS 4 after treatment. Next, using GO Biological Process pathway analysis we examined the top 20 pathways statistically significantly altered by therapy (Supplemental Figure 8D). Not surprisingly, many of these pathways reflect changes in cell metabolic processes and are likely related to gene expression changes in cancer cells and stroma. However, the third most highly significant pathway is regulation of adaptive immune response suggesting that treatment is altering gene expression of immune pathways within the tumor microenvironment. Also, as outlined above (Supplemental Figure 5-6), gene expression analysis corroborated the changes in immune cell populations in the tumor microenvironment identified by IF. We further analyzed the paired biopsy samples based on HFRT vs LDFRT treatment groups with 2 paired biopsies in each sample. While conclusions are limited by the small sample size some interesting hypothesis generating findings were noted. In comparing HFRT versus LDFRT there are 990 differentially expressed genes in the HFRT cohort and only 147 differentially expressed genes in the LDFRT suggesting that HFRT + dual ICI causes more gene expression changes in the tumor microenvironment than LDFRT + dual ICI. Evaluation of gene marker sets of different immune cell types in the tumor microenvironment using GSVA revealed no statistically significant therapy induced changes in the gene marker sets for any immune cell type in the LDFRT or HFRT samples other than the changes already noted above (Supplemental Figure $5-6)$.

As both immune checkpoint inhibitors and radiotherapy have been reported to have their most pronounced effects on T-cells, we next examined therapy induced differences in expression of T-cell related genes in circulating PBMCs. Hierarchical clustering based upon expression of T-cell activation genes (GO Biological Processes) revealed separation of preand post- therapy samples (Figure 5B). KEGG biological pathways also identified T-cell signaling as a major pathway impacted by treatment (Figure 5C), which was also evident by principal component analysis (PCA) of the antigen receptor-mediated signaling gene expression data (Figure 5E). These data suggest therapy induced differences in T-cell functionality, at least at a transcriptional level. Analysis of the individual T-cell activation genes revealed significant therapy-associated declines in gene expression (Figure 5D). These findings are likely due to an overall post-treatment decline in T-cells (Figure 4A-C). Overall, 
these results corroborate the significant declines in T-cell populations observed by CyTOF analysis in Figure 4.

\section{Treatment was associated with alterations of the tumor-infiltrating and peripheral $\mathrm{T}$ cell} repertoires

As another means to evaluate treatment-associated immune alterations, we quantified the number of expanded and contracted clones in tumor and blood following therapy. CDR3 sequence analysis revealed a therapy-associated increase in the number of expanded compared to contracted T cell clones in PBMCs. In contrast, T-cell expansions were also noted in the tumor microenvironment, but these were accompanied by a comparable number of T-cell contractions (Figure 5G). To better visualize the T-cell clonal dynamics following therapy, we constructed galaxy plots of the PBMC and tumor microenvironment T-cell repertoires, in which the number of clones, anatomical location, and CDR3 sequence similarities were represented by the size, color, and location of the plotted circles, respectively (Figure 5H). On these plots, expansions and contractions within the tumor and PBMCs can be visualized following treatment. Clusters within the plots are formed when unique T-cell clones have similar CDR3 TCR sequences, presumably due to shared antigen specificity. Thus, some of the new treatment-induced clonal expansions that appeared in the tumor had similar TCR sequences. The overlap between the tumor-infiltrating T-cell repertoire and the peripheral T-cell repertoire is also clearly evident, with each anatomical site having both shared and unique clones (Figure 5H). The Shannon diversity of the T-cell repertoire pre and post-therapy was then calculated to help characterize the treatmentassociated changes. In the PBMC population there was a significant decrease in Shannon diversity (Figure 5I), which is likely due to the noted increase in T-cell expansions (Figure 5G). Finally, pie charts were also constructed, which revealed similar treatment-induced alterations in the T-cell repertoire, with several new T-cell expansions appearing following therapy (Figure 5J).

\section{Discussion}

In this phase 2 trial, we found that the PD-L1 inhibitor durvalumab and CTLA-4 inhibitor tremelimumab combined with either HFRT or LDFRT directed against 1-2 liver metastases was tolerated with the spectrum and frequency of immune-related adverse events expected from combined PD-1/L1, CTLA-4 blockade alone (2). No unexpected radiation-related adverse events were observed. These results demonstrate the feasibility of combining PDL1/CTLA-4 blockade with focused liver-directed radiation for patients with metastatic colorectal cancer and other malignancy types.

Despite promising preclinical data demonstrating radiation can induce tumor-specific immune responses (18) and increased T-cell infiltration (6) and immune-mediated tumor cell death,(19) clinical trials to date have demonstrated mixed results. Translation to the clinic has been hindered by uncertainty regarding the appropriate radiation dose to induce immune modulation.(20) In preclinical models, a variety of different radiation dose / fractionation regimens have been demonstrated to induce interferon-stimulated genes via generation of micronuclei / primary nuclear rupture leading to cytosolic dsDNA and activation of the 
cGAS/STING pathway.(21-23) The dose threshold for this effect is uncertain, particularly in human tumors. This is a relevant clinical question, as higher radiation doses are not only associated with increased side effects but may also lead to regulatory inhibition of the cGAS/STING pathway (24) and deleterious lymphopenia.(25) Lower doses of radiation have also been associated with other positive immune effects, in particular inducing a more favorable presence of M1 macrophages.(6)

We did not observe objective responses outside of the radiation treatment field in either the HFRT and LDFRT arms, and median progression-free and overall survival was limited. These results are likely due, at least in part, to the advanced nature of disease and extensive pretreatment among enrolled patients given the median number of prior lines of therapy was 4 (range 1-7). In contrast to earlier, untreated CRC patients treated with immunotherapy alone prior to any other treatment,(26) these patients' immune systems may be impaired by advanced tumor burden and prior treatment;(27) and may thus be less likely to respond to combined immune checkpoint / radiation strategies. Indeed, combination radiation / immune checkpoint blockade treatment strategies may have more promise in earlier disease settings(28-31). This approach should be tested in patients with less extensive disease burden, or perhaps oligometastatic CRC disease to the liver - a setting in which all gross disease can potentially be targeted by ablative radiation treatment(31). Additionally, we found relatively low PD-L1 expression across tumor specimens, with the highest expression of PD-L1 observed in the patient with evidence of an out of field response in a porta hepatis lymph node. Thus, this low PD-L1 expression may contribute to the lack of benefit. Overall, our results clearly demonstrated that at the selected radiation doses / schedules and in this particular clinical setting, focal radiation did not reverse resistance to anti-PD-L1 / CTLA-4 in a tumor type resistant to $\mathrm{PD}(\mathrm{L})-1 / \mathrm{CTLA}-4$ directed therapy.

We explored biomarkers such as PD-L1 tumor expression and infiltrating CD8+ T-cells predictive of response to immune checkpoint blockade in other studies. In line with previous studies,(1) PD-L1 expression was generally low or negative in this cohort of MSS colorectal cancer, and CD8+ T-cell infiltration was variable. We did observe the highest levels of PDL1 expression, CD8+ T-cell infiltration and PD-1+ cell infiltration in the pretreatment irradiated liver lesion of one patient with stable disease on study and a notable out-of-field response. Of interest, this patient had targeted next-generation tumor sequencing that revealed a POLE mutation of questionable functional significance (c.1784A>G (p.N595S), exon 16 - in $91 \%$ of 343 reads). POLE mutations have been associated with higher mutational burden in MSS colorectal cancers and may predispose for response to immune checkpoint blockade. (32) Interestingly, we also observed a non-significant trend towards greater CD4+ and CD8+ T-cell infiltration in patients who previously received radiation treatment, but these comparisons were limited by small numbers in the prior radiation group.

Despite the lack of systemic objective responses, correlative studies performed on matched tumor biopsies taken before and after therapy suggest that this combination therapy alters the tumor immune microenvironment. We observed increases in CD8+, and proliferating CD8+/PD1+/Ki67+ T-cells after HFRT in comparison to LDFRT. Gene expression analyses supported this finding. Higher dose radiation has been associated with immunogenic cell death with increased cell-surface calreticulin, and increased HMGB1 and ATP release, (19) 
that might be associated with greater levels of immune activation and increased T-cell infiltration over time.

Immunologic impacts of LDFRT in human tumors were more uncertain given these regimens are not standardly used in clinical practice and less likely to result in tumor cell death in the absence of systemic therapy. Within the local tumor microenvironment, we observed modest increases in CD8+ T-cell infiltration and variable changes in the CD8+/ PD1+/Ki67+ subset following LDFRT. However, consistent with preclinical data,(6) LDFRT led to increases in the M1/M2 macrophage ratio in all 3 LDFRT patients as compared to none of the HFRT patients. In the HFRT patients the M1/M2 macrophage ratio actually decreases, which is also consistent with preclinical reports that demonstrate an increase in M2 macrophages after RT at higher doses (pmid: 24992164, 26946344). Gene expression data also showed an increase of macrophage gene marker sets post therapy in the HFRT patients but clear conclusions could not be drawn regarding expression changes of macrophage polarization genes. Drawing conclusions from these samples is difficult given the limited sample size and that RNAseq was bulk RNAseq which can limit detecting changes in cell types which are scarce in comparison to tumor and stromal cells. Intriguingly, we also observed pronounced increases in micronuclei / primary nuclear rupture with LDFRT. Previous studies have highlighted the importance of these structures in leading to cGAS/STING activation following radiation and subsequent immune response. $(22,23)$ The ability of low-dose radiation to generate micronuclei / primary nuclear rupture in combination with immune checkpoint blockade in human tumors is intriguing and could be explored in future studies. The failure to observe micronuclei / primary nuclear rupture after HFRT may have been a result of the 7-8 week timepoint that was evaluated, as cells with multiple abnormal nuclear structures including multiple micronuclei, chromosome bridges and primary nuclear rupture may have been selected against over time because of limited proliferation capacity.

Evaluation of circulating biomarkers demonstrated that both treatment regimens led to systemic immunologic changes. Olink serum profiling demonstrated that in both radiation immunotherapy arms there was evidence of increased interferon-gamma and related chemokines such as CXCL-9, 10, and 11. We observed post treatment changes in circulating PD-L1, and these are likely due to the ligand being stabilized as a result of durvalumab treatment. T-cell receptor sequencing identified some specific T-cell clones expanded in the tumor microenvironment as well as the peripheral circulation, as has been observed following radiation and immune checkpoint blockade in a prior study evaluating the combination of ipilimumab and hypofractionated radiation in NSCLC.(29) Both treatment regimens led to non-significant overall declines in absolute lymphocyte count that was more pronounced in patients that received HFRT, as would be expected from prior studies demonstrating the potential lymphopenia that can be induced by higher dose radiation.(25) More unexpected were significant declines observed in multiple circulating activated T-cell subsets in patients treated with HFRT as compared with LDFRT, again supported by gene expression data. It is possible that these declines in circulating effector T-cell populations limited systemic response despite local immune activation. Interestingly, LDFRT led to declines in specific circulating populations of regulatory T-cells. 
Limitations of our study include the choice of two specific HFRT and LDFRT regimens and timing in relation to immune checkpoint blockade, and the decision to irradiate 1-2 metastatic liver lesions as opposed to more lesions or metastases elsewhere in the body. These aspects of our study design helped maintain homogeneity, but it is possible that different radiation timing or parameters may be more conducive to generating an effective systemic immune response. In terms of correlative data, limited numbers of study patients had matched pre- and post-biopsy tissue available for study, in part because several patients had no viable tumor at the irradiated site at the time of repeat biopsy, making it difficult to discern local treatment effects. Therefore, the immune changes we observed following immune checkpoint blockade and both HFRT and LDFRT are exploratory, although the formation of micronuclei/primary nuclear rupture, increases in CD8+ T-cell subsets after HFRT and increase in M1/M2 macrophage ratios are all concordant with preclinical data.

In summary, although the combination of HFRT and LDFRT did not lead to any unexpected toxicities, we did not observe objective responses outside the radiation treatment field, suggesting irradiating 1-2 lesions at these radiation dose/fractionations in combination with immune checkpoint blockade is not sufficient to mediate systemic antitumor immunity in refractory colorectal cancer patients. We did observe local immunologic changes following both HFRT and LDFRT, although the nature and extent of changes appeared different between the two radiation schedules. Our biomarker findings generated interesting hypotheses for further testing. Future efforts should focus on increasing the positive immunologic impacts of focused radiation therapy, perhaps by irradiating more lesions such as in the case of oligometastatic or oligoprogressive disease,(31) or perhaps by investigating LDFRT in earlier disease settings, while also attempting to minimize any potential detrimental immune effects of irradiation such as radiation-induced declines in circulating activated T-cell populations.

\section{Supplementary Material}

Refer to Web version on PubMed Central for supplementary material.

\section{Acknowledgments:}

We acknowledge Janice Russell and all participating patients and sites. We would also like to thank John Daley and his team at the Longwood Medical Area CyTOF Core at DFCI for their technical assistance.

Funding: The trial was conducted under auspices of the Experimental Therapeutics Clinical Trials Network (ETCTN) and funded by UM1 CA186709 (Geoffrey Shapiro, PI), a Biomarker Supplement to UM1 CA186709 (JDS, SJR and SFH, Project Leaders), and Center for Immuno-Oncology, Dana-Farber Cancer Institute. Scientific and financial support for the CIMAC-CIDC Network is provided through the National Cancer Institute (NCI) Cooperative Agreements U24CA224319 (to the Icahn School of Medicine at Mount Sinai CIMAC), U24CA224331 (to the Dana-Farber Cancer Institute CIMAC), and U24CA224316 (to the CIDC at Dana-Farber Cancer Institute). Additional support is made possible through the NCI CTIMS Contract HHSN261201600002C. Scientific and financial support for the PACT project are made possible through funding support provided to the FNIH by: AbbVie Inc., Amgen Inc., Boehringer-Ingelheim Pharma GmbH \& Co. KG., Bristol-Myers Squibb, Celgene Corporation, Genentech Inc, Gilead, GlaxoSmithKline plc, Janssen Pharmaceutical Companies of Johnson \& Johnson, Novartis Institutes for Biomedical Research, Pfizer Inc., and Sanofi. The CIMAC-CIDC website is found at https://cimacnetwork.org/.

Competing interests: AMM reports research support paid to the institution and/or advisory board fees: Merck, BMS, AstraZeneca, Genentech, EMD Serono, Zosano, Incyte, Dynavax. Expert witness fees. AL is a current employee of BMS. EMT reports travel fees and honorariums from Fluidigm Corporation and is a current Fluidigm 
employees. RDG reports consulting fees for AstraZeneca, Pfizer, and BluePrint Medicines. Research support to institution from Pfizer, Merck, Bristol-Myers Squibb, Jounce, Helsinn, Takeda. OBA reports research support from BMS, Taiho, Ipsen, GSK, Calithera and SynCore Biotechnology; and received advisory board fees from Conjupro, Ipsen, R-Pharma and Natera. OER reports research support from Merck. Speaker for activities supported by educational grants from BMS and Merck. Consultant for Merck, Celgene, Five Prime, GSK, Bayer, Roche/ Genentech, Puretech, Imvax, Sobi. Pending patent "Methods of using pembrolizumab and trebananib." JMC received research funding to his institution from Abbvie, Merus, Roche, and Bristol Myers Squib. He received research funding from Merck, Astrazeneca, Esperas Pharma, and Tesaro, received consulting fees from Bristol Myers Squibb, and received travel funding from Bristol Myers Squib. HJM reports honorarium from Merck. M.C. serves as a consultant and has honoraria and/or research funding from Amgen, Taiho, Astella, Ipsen, BMS, QED, Incyte Eisai, AstraZeneca, Pfizer and Exelixis. SKJ reports research funding, consultant, and personal fees from Merck. Consultant for Syntactx. SG reports consultancy and/or advisory roles for Merck, Neon Therapeutics and OncoMed and research funding from Bristol-Myers Squibb, Genentech, Immune Design, Agenus, Janssen R\&D, Pfizer, Takeda and Regeneron. S.G. was supported by grants U24 CA224319, U01 DK124165, P01 CA190174, and DOD W81XWH-18-1-0528. SJR receives research support from Merck, Bristol Myers Squibb, Affimed, and KITE/ Gilead. He is on the Scientific Advisory Boards for Immunitas and RareCyte.FSH reports grants, personal fees and other from Bristol-Myers Squibb, personal fees from Merck, personal fees from EMD Serono, grants, personal fees and other from Novartis, personal fees from Surface, personal fees from Compass Therapeutics, personal fees from Apricity, personal fees from Aduro, personal fees from Sanofi, personal fees from Pionyr, personal fees from 7 Hills Pharma, personal fees from Verastem, personal fees from Torque, personal fees from Rheos, personal fees from Kairos, personal fees from Bicara, from Psioxus Therapeutics, other from Pieris Pharmacutical, from Zumutor, from Corner Therapeutics, personal fees from Eisai, personal fees from Checkpoint Therapeutics, outside the submitted work; In addition, he has a patent Methods for Treating MICA-Related Disorders (\#20100111973) with royalties paid, a patent Tumor antigens and uses thereof (\#7250291) issued, a patent Angiopoiten-2 Biomarkers Predictive of Anti-immune checkpoint response (\#20170248603) pending, a patent Compositions and Methods for Identification, Assessment, Prevention, and Treatment of Melanoma using PD-L1 Isoforms (\#20160340407) pending, a patent Therapeutic peptides (\#20160046716) pending, a patent Therapeutic Peptides (\#20140004112) pending, a patent Therapeutic Peptides (\#20170022275) pending, a patent Therapeutic Peptides (\#20170008962) pending, a patent THERAPEUTIC PEPTIDES

Therapeutic Peptides. Patent number: 9402905 issued, a patent METHODS OF USING PEMBROLIZUMAB AND TREBANANIB pending, a patent Vaccine compositions and methods for restoring NKG2D pathway function against cancers Patent number: 10279021 issued, a patent Antibodies that bind to MHC class I polypeptide-related sequence $\mathrm{A}$

Patent number: 10106611 issued, and a patent ANTI-GALECTIN ANTIBODY BIOMARKERS PREDICTIVE OF ANTI-IMMUNE CHECKPOINT AND ANTI-ANGIOGENESIS RESPONSES Publication number: 20170343552 pending. JDS reports research support paid to the institution: Merck, BMS, Regeneron, Debiopharm. Consulting / Scientific Advisory Board / Travel fees: Debiopharm, BMS, Nanobiotix, Tilos, AstraZeneca, LEK, Catenion, ACI Clinical, Immunitas, STIMIT, Astellas. Expert witness fees.

\section{References}

1. Le DT, Uram JN, Wang H, Bartlett BR, Kemberling H, Eyring AD, et al. PD-1 Blockade in Tumors with Mismatch-Repair Deficiency. The New England journal of medicine 2015;372(26):2509-20 doi 10.1056/NEJMoa1500596. [PubMed: 26028255]

2. Overman MJ, McDermott R, Leach JL, Lonardi S, Lenz HJ, Morse MA, et al. Nivolumab in patients with metastatic DNA mismatch repair-deficient or microsatellite instability-high colorectal cancer (CheckMate 142): an open-label, multicentre, phase 2 study. The Lancet Oncology 2017;18(9):1182-91 doi 10.1016/S1470-2045(17)30422-9. [PubMed: 28734759]

3. Ngwa W, Irabor OC, Schoenfeld JD, Hesser J, Demaria S, Formenti SC. Using immunotherapy to boost the abscopal effect. Nat Rev Cancer 2018 doi 10.1038/nrc.2018.6.

4. Twyman-Saint Victor C, Rech AJ, Maity A, Rengan R, Pauken KE, Stelekati E, et al. Radiation and dual checkpoint blockade activate non-redundant immune mechanisms in cancer. Nature 2015;520(7547):373-7 doi 10.1038/nature14292. [PubMed: 25754329]

5. Dewan MZ, Galloway AE, Kawashima N, Dewyngaert JK, Babb JS, Formenti SC, et al. Fractionated but not single-dose radiotherapy induces an immune-mediated abscopal effect when combined with anti-CTLA-4 antibody. Clinical cancer research : an official journal of the American Association for Cancer Research 2009;15(17):5379-88 doi 10.1158/1078-0432.CCR-09-0265. [PubMed: 19706802] 
6. Klug F, Prakash H, Huber PE, Seibel T, Bender N, Halama N, et al. Low-Dose Irradiation Programs Macrophage Differentiation to an iNOS(+)/M1 Phenotype that Orchestrates Effective T Cell Immunotherapy. Cancer Cell. Volume 242013. p 589-602.

7. Arnold SM, Regine WF, Ahmed MM, Valentino J, Spring P, Kudrimoti M, et al. Low-dose fractionated radiation as a chemopotentiator of neoadjuvant paclitaxel and carboplatin for locally advanced squamous cell carcinoma of the head and neck: results of a new treatment paradigm. International journal of radiation oncology, biology, physics 2004;58(5):1411-7 doi 10.1016/ j.ijrobp.2003.09.019.

8. Reiss KA, Herman JM, Zahurak M, Brade A, Dawson LA, Scardina A, et al. A Phase I study of veliparib (ABT-888) in combination with low-dose fractionated whole abdominal radiation therapy in patients with advanced solid malignancies and peritoneal carcinomatosis. Clinical cancer research : an official journal of the American Association for Cancer Research 2015;21(1):68-76 doi 10.1158/1078-0432.CCR-14-1552. [PubMed: 25355929]

9. Eisenhauer EA, Therasse P, Bogaerts J, Schwartz LH, Sargent D, Ford R, et al. New response evaluation criteria in solid tumours: revised RECIST guideline (version 1.1). Eur J Cancer 2009;45(2):228-47 doi 10.1016/j.ejca.2008.10.026. [PubMed: 19097774]

10. National Cancer Institute DoCTaD, Cancer Therapy Evaluation Program. <https://ctep.cancer.gov/ protocoldevelopment/electronic_applications/ctc.htm\#ctc_40>.

11. Griffin GK, Weirather JL, Roemer M, Lipschitz M, Kelley A, Chen PH, et al. Spatial Signatures Identify Immune Escape via PD-1 as a Defining Feature of T-cell/Histiocyte-rich Large B-cell Lymphoma. Blood 2020 doi 10.1182/blood.2020006464.

12. Patel SS, Weirather JL, Lipschitz M, Lako A, Chen PH, Griffin GK, et al. The microenvironmental niche in classic Hodgkin lymphoma is enriched for CTLA-4-positive T cells that are PD-1negative. Blood 2019;134(23):2059-69 doi 10.1182/blood.2019002206. [PubMed: 31697809]

13. Carey CD, Gusenleitner D, Lipschitz M, Roemer MGM, Stack EC, Gjini E, et al. Topological analysis reveals a PD-L1-associated microenvironmental niche for Reed-Sternberg cells in Hodgkin lymphoma. Blood 2017;130(22):2420-30 doi 10.1182/blood-2017-03-770719. [PubMed: 28893733]

14. Kleinsteuber K, Corleis B, Rashidi N, Nchinda N, Lisanti A, Cho JL, et al. Standardization and quality control for high-dimensional mass cytometry studies of human samples. Cytometry A 2016;89(10):903-13 doi 10.1002/cyto.a.22935. [PubMed: 27575385]

15. Thrash EM, Kleinsteuber K, Hathaway ES, Nazzaro M, Haas E, Hodi FS, et al. High-Throughput Mass Cytometry Staining for Immunophenotyping Clinical Samples. STAR Protocols 2020:100055 doi 10.1016/j.xpro.2020.100055. [PubMed: 33111099]

16. Dillen CA, Pinsker BL, Marusina AI, Merleev AA, Farber ON, Liu H, et al. Clonally expanded gammadelta $\mathrm{T}$ cells protect against Staphylococcus aureus skin reinfection. J Clin Invest 2018;128(3):1026-42 doi 10.1172/JCI96481. [PubMed: 29400698]

17. Guinney J, Dienstmann R, Wang X, de Reynies A, Schlicker A, Soneson C, et al. The consensus molecular subtypes of colorectal cancer. Nature medicine 2015;21(11):1350-6 doi 10.1038/ nm.3967.

18. Sharabi AB, Nirschl CJ, Kochel CM, Nirschl TR, Francica BJ, Velarde E, et al. Stereotactic Radiation Therapy Augments Antigen-Specific PD-1-Mediated Antitumor Immune Responses via Cross-Presentation of Tumor Antigen. Cancer immunology research 2015;3(4):345-55 doi 10.1158/2326-6066.CIR-14-0196. [PubMed: 25527358]

19. Golden EB, Frances D, Pellicciotta I, Demaria S, Helen Barcellos-Hoff M, Formenti SC. Radiation fosters dose-dependent and chemotherapy-induced immunogenic cell death. Oncoimmunology 2014;3:e28518 doi 10.4161/onci.28518. [PubMed: 25071979]

20. Monjazeb AM, Schoenfeld JD. Radiation dose and checkpoint blockade immunotherapy: unanswered questions. The Lancet Oncology 2016;17(1):e3-4 doi 10.1016/ S1470-2045(15)00541-0. [PubMed: 26758758]

21. Deng L, Liang H, Xu M, Yang X, Burnette B, Arina A, et al. STING-Dependent Cytosolic DNA Sensing Promotes Radiation-Induced Type I Interferon-Dependent Antitumor Immunity in Immunogenic Tumors. Immunity 2014;41(5):843-52 doi 10.1016/j.immuni.2014.10.019. [PubMed: 25517616] 
22. Harding SM, Benci JL, Irianto J, Discher DE, Minn AJ, Greenberg RA. Mitotic progression following DNA damage enables pattern recognition within micronuclei. Nature 2017;548(7668):466-70 doi 10.1038/nature23470. [PubMed: 28759889]

23. Mackenzie KJ, Carroll P, Martin CA, Murina O, Fluteau A, Simpson DJ, et al. cGAS surveillance of micronuclei links genome instability to innate immunity. Nature 2017;548(7668):461-5 doi 10.1038/nature23449. [PubMed: 28738408]

24. Vanpouille-Box C, Alard A, Aryankalayil MJ, Sarfraz Y, Diamond JM, Schneider RJ, et al. DNA exonuclease Trex 1 regulates radiotherapy-induced tumour immunogenicity. Nature communications 2017;8:15618 doi 10.1038/ncomms15618.

25. Pike LRG, Bang A, Mahal BA, Taylor A, Krishnan M, Spektor A, et al. The Impact of Radiation Therapy on Lymphocyte Count and Survival in Metastatic Cancer Patients Receiving PD-1 Immune Checkpoint Inhibitors. International journal of radiation oncology, biology, physics 2019;103(1):142-51 doi 10.1016/j.ijrobp.2018.09.010.

26. Chalabi M, Fanchi LF, Dijkstra KK, Van den Berg JG, Aalbers AG, Sikorska K, et al. Neoadjuvant immunotherapy leads to pathological responses in MMR-proficient and MMR-deficient earlystage colon cancers. Nature medicine 2020 doi 10.1038/s41591-020-0805-8.

27. Huang AC, Postow MA, Orlowski RJ, Mick R, Bengsch B, Manne S, et al. T-cell invigoration to tumour burden ratio associated with anti-PD-1 response. Nature 2017;545(7652):60-5 doi 10.1038/nature22079. [PubMed: 28397821]

28. Antonia SJ, Villegas A, Daniel D, Vicente D, Murakami S, Hui R, et al. Overall Survival with Durvalumab after Chemoradiotherapy in Stage III NSCLC. The New England journal of medicine 2018 doi 10.1056/NEJMoa1809697.

29. Formenti SC, Rudqvist NP, Golden E, Cooper B, Wennerberg E, Lhuillier C, et al. Radiotherapy induces responses of lung cancer to CTLA-4 blockade. Nature medicine 2018;24(12):1845-51 doi 10.1038/s41591-018-0232-2.

30. Theelen W, Peulen HMU, Lalezari F, van der Noort V, de Vries JF, Aerts J, et al. Effect of Pembrolizumab After Stereotactic Body Radiotherapy vs Pembrolizumab Alone on Tumor Response in Patients With Advanced Non-Small Cell Lung Cancer: Results of the PEMBRO-RT Phase 2 Randomized Clinical Trial. JAMA oncology 2019 doi 10.1001/jamaoncol.2019.1478.

31. Bauml JM, Mick R, Ciunci C, Aggarwal C, Davis C, Evans T, et al. Pembrolizumab After Completion of Locally Ablative Therapy for Oligometastatic Non-Small Cell Lung Cancer: A Phase 2 Trial. JAMA oncology 2019 doi 10.1001/jamaoncol.2019.1449.

32. Wang F, Zhao Q, Wang YN, Jin Y, He MM, Liu ZX, et al. Evaluation of POLE and POLD1 Mutations as Biomarkers for Immunotherapy Outcomes Across Multiple Cancer Types. JAMA Oncol 2019 doi 10.1001/jamaoncol.2019.2963. 


\section{Statement of Translational Relevance:}

Microsatellite stable colorectal cancers are resistant to immune checkpoint blockade with PD-1/L1 and/or CTLA-4 inhibitors. Focal radiation therapy has been demonstrated to impact anti-tumor immunity in preclinical models, but the impact of different radiation doses in human tumors is uncertain. We conducted a randomized phase II study evaluating the combination of the PD-L1 inhibitor durvalumab and the CTLA-4 inhibitor tremelimumab with either hypofractionated radiation (HFRT), or low-dose fractionated radiation (LDFRT) in patients with advanced MSS CRC. Despite tolerability of both regimens, HFRT or LDFRT given with combined PD-L1/CTLA-4 inhibition to metastatic colorectal patients did not lead to systemic response but was associated with local and systemic immunologic changes including T-cell infiltration, formation of micronuclei / primary nuclear rupture, and changes in circulating T-cell populations and T-cell diversity. These findings inform future trials to optimize radiation parameters to capitalize on immune stimulation. 

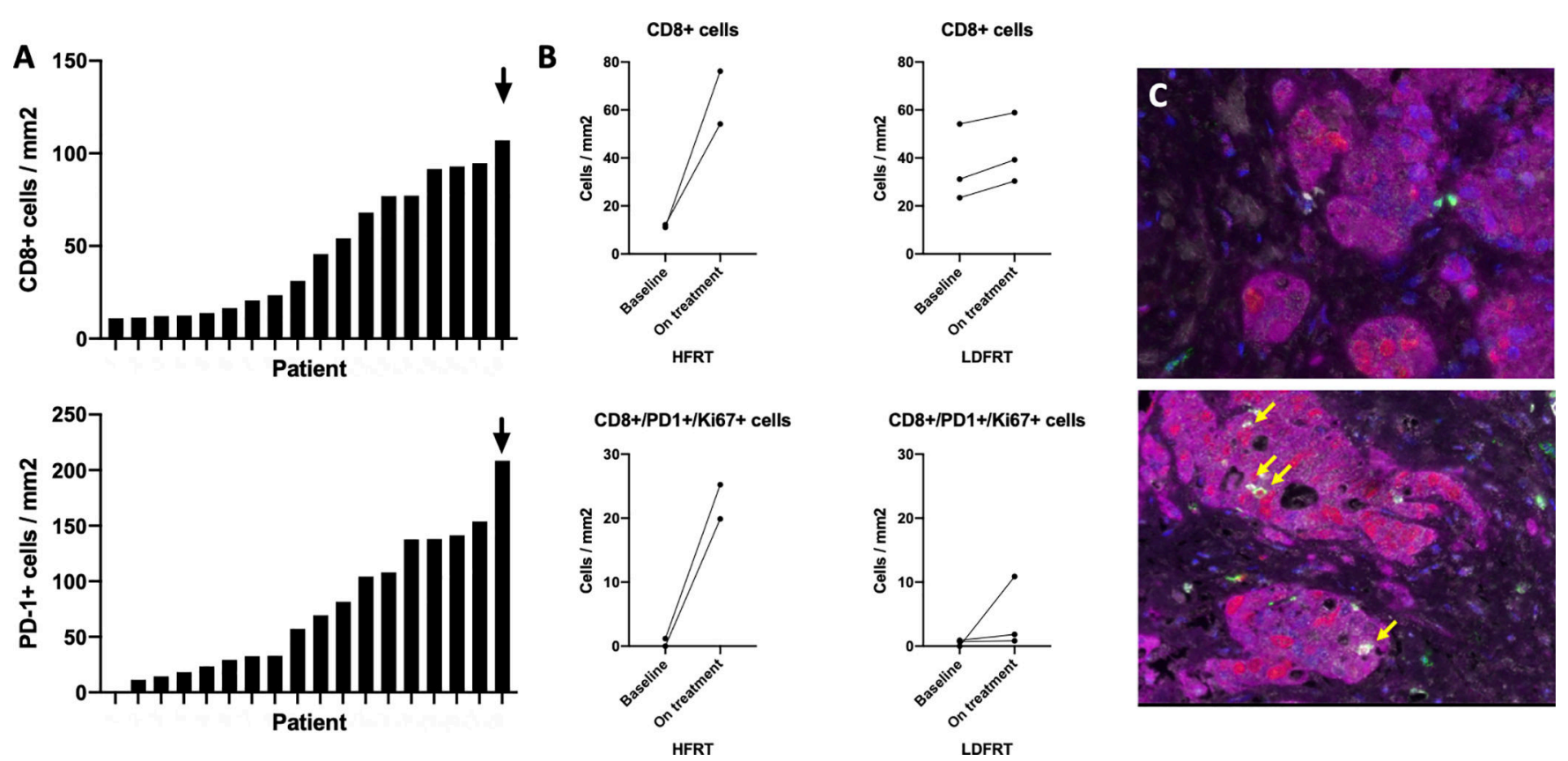

Figure 1.

T-cell populations in the tumor microenvironment. Multiplex immunofluorescence evaluating expression of cyokeratin (purple), CD8 (white), PD-1 (green) and Ki67 (red) as shown on the right. Baseline variability in CD8+ and PD-1+ cell populations (A), with highest levels of both populations observed in the irradiated liver lesion from the subject who reported an out-of-field response. Changes in cell populations $(\mathbf{B})$ over the course of treatment in the hypofractionated radiation (HFRT) and low dose fractionated radiation therapy (LDFRT) arms (on-treatment samples obtained week 7-8). In 1B, for each patient, the variance at a time point was estimated by [number of frames * SEM2] and the variance of the difference (post-pre) was estimated by the sum of the time point variances. The standard deviation of the difference was the square root of the variance. Baseline (top) and on treatment (bottom) specimen from a patient treated with HFRT (C) demonstrates increases in CD8+/PD1+/Ki67+ cells (yellow arrows). 


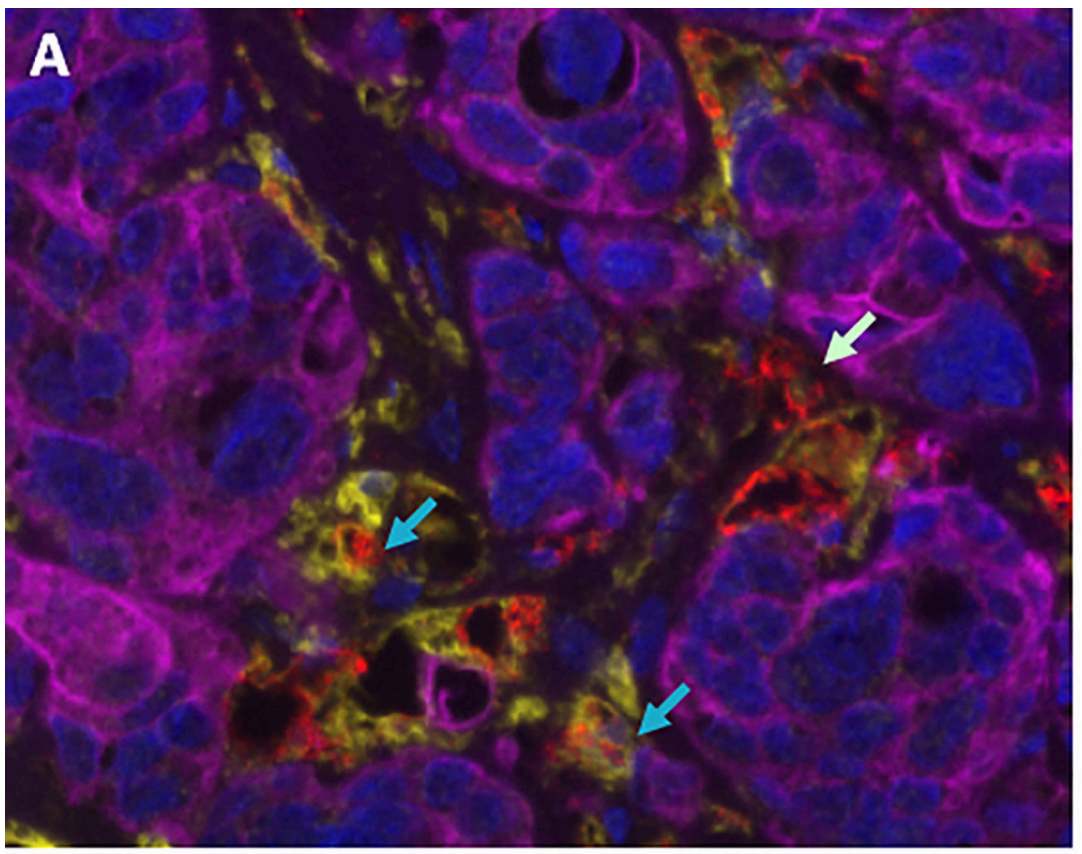

B

M1 / M2 Macrophage Ratio M1 / M2 Macrophage Ratio
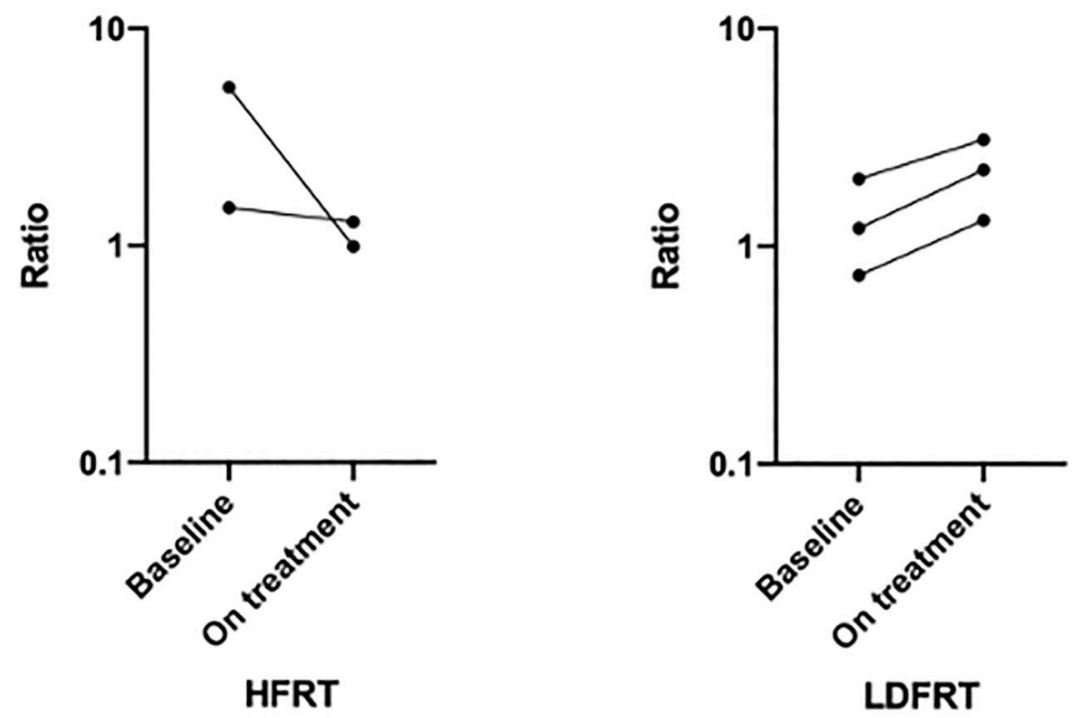

Figure 2.

Macrophage populations within the tumor microenvironment. Multiplex immunofluorescence evaluating expression of cyokeratin (purple), DAPI (blue), CD68 (red) and CD163 (yellow) as shown (A). M1 macrophages demonstrate CD68 staining (white arrow) while M2 demonstrate CD68 and CD163 co staining (blue arrows). Changes in the ratio of M1 to M2 cell populations over the course of treatment (on-treatment samples obtained week 7-8) in the hypofractionated radiation (HFRT) and low dose fractionated radiation therapy (LDFRT) arms $(\mathbf{B})$. 

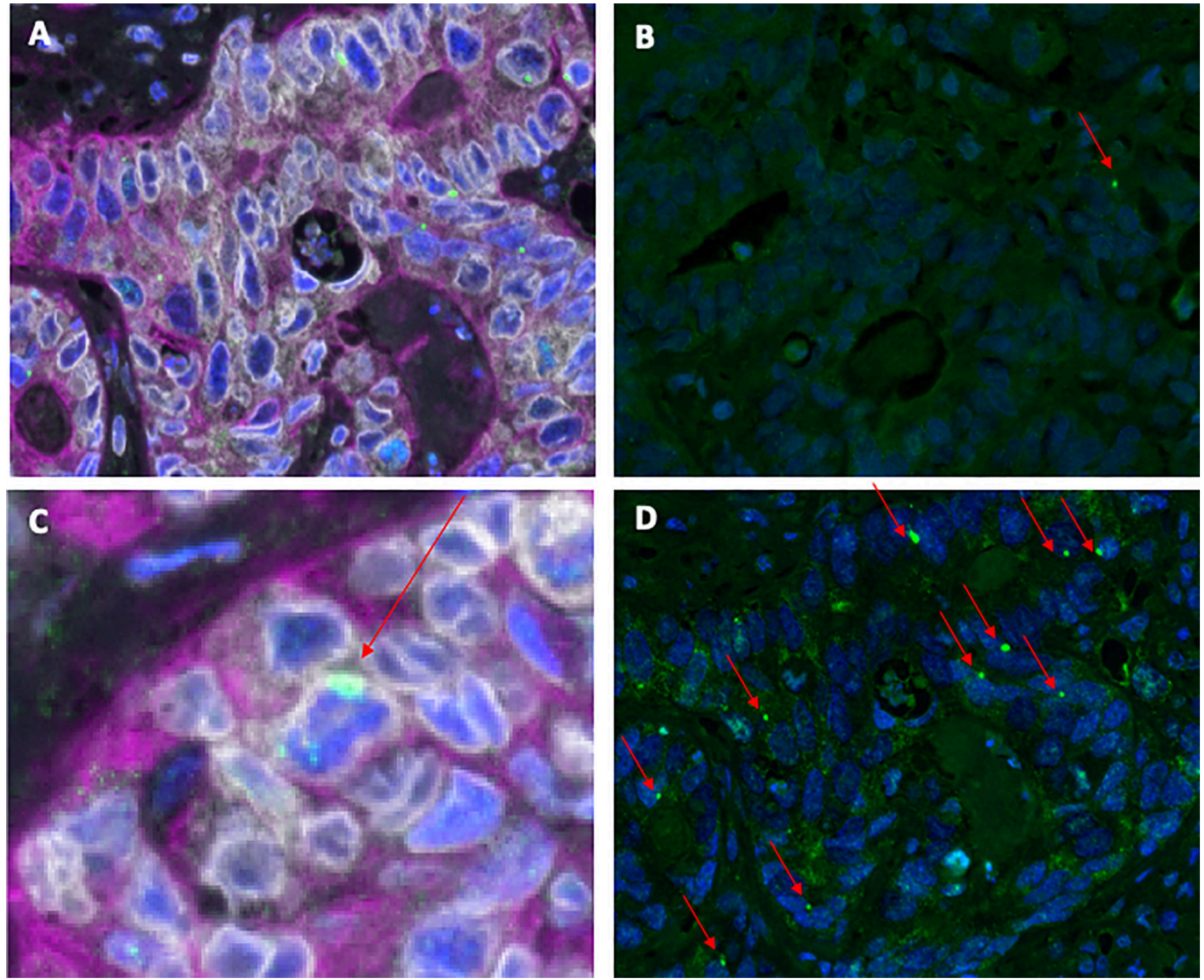

Figure 3.

Multiplex immunofluorescence evaluating formation of micronuclei and primary nuclear ruptures. Samples were stained for cytokeratin (purple), DAPI (blue), cGAS (green) and Lamin B receptor (white) as shown (low power, A). Micronuclei and primary nuclear rupture(s) were scored by identifying co-localization of cGAS and DAPI outside of the nucleus for micronuclei and cGAS with a DAPI defect in the nuclear rim of primary nuclei for primary nuclear ruptures (red arrow, B). Pre-treatment (B) and post-treatment (D) specimens (on-treatment samples obtained week 7-8) demonstrate pronounced increase in micronuclei and foci of primary nuclear ruptures (red arrows) in 2 subjects. 

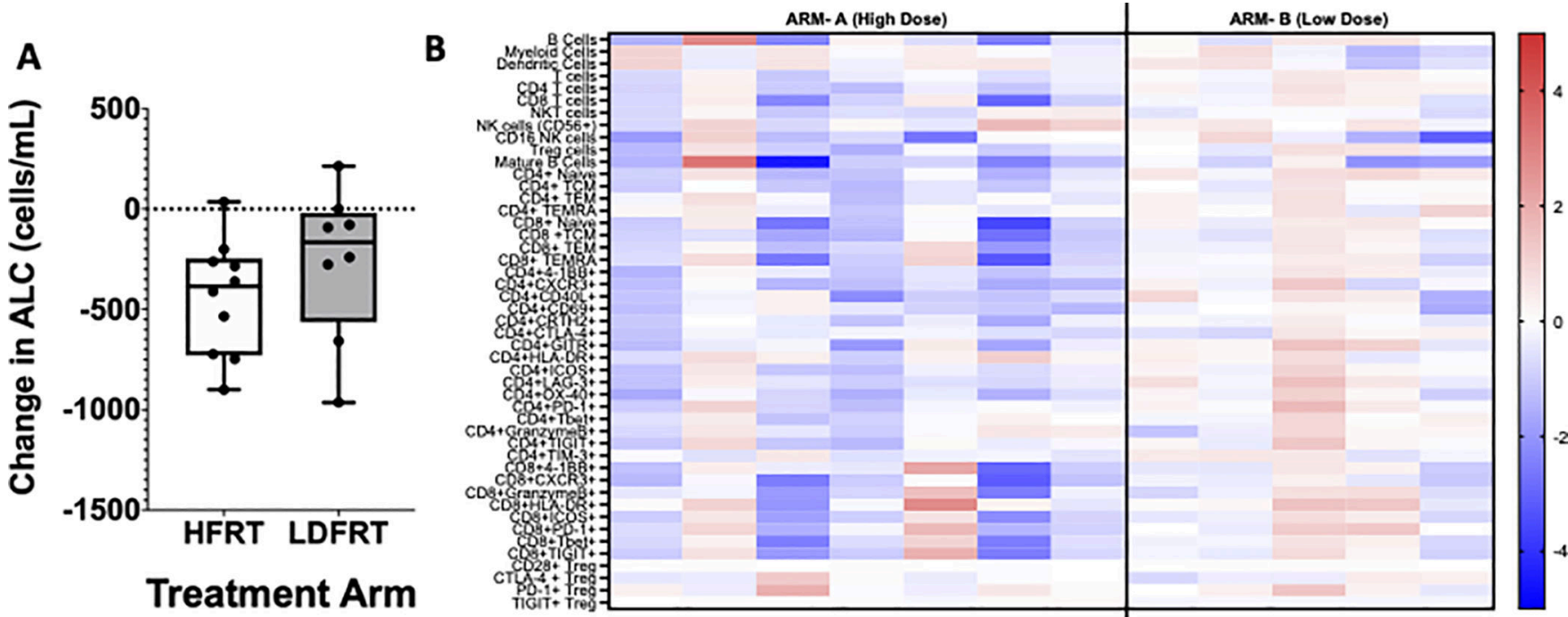

C

CD4 and CD8 Activated Cell Populations

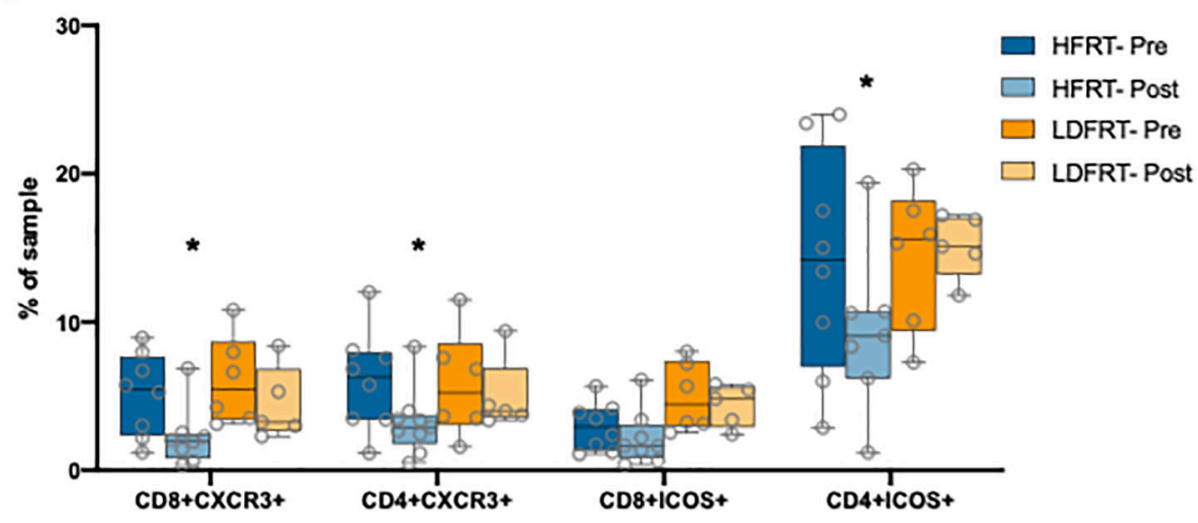

Figure 4.

Changes in circulating biomarkers between pre-treatment and week 5 of treatment-weeks. Absolute lymphocyte count (ALC, cells $/ \mathrm{mL})(\mathbf{A})$. Median, inter-quartile range and maximum changes are plotted for both treatment arms. Fold-change in cell populations over the course of treatment $(\mathbf{B}, \mathbf{C})$. Heat map shows $\log 2$ fold changes for each patient. Significant changes $(\mathrm{p}<0.05)$ denoted with *. 
A

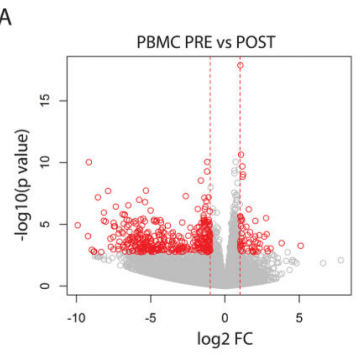

B

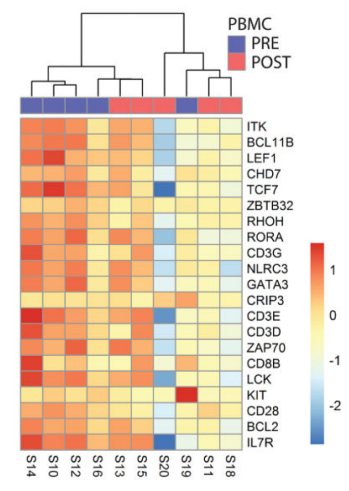

$\mathrm{F}$
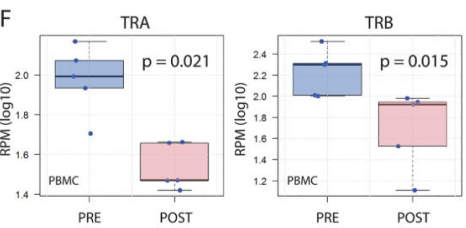

C

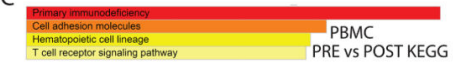

D PBMC
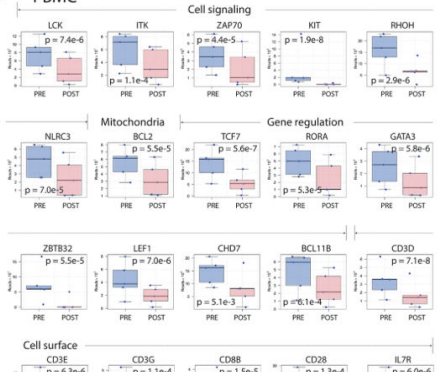

-

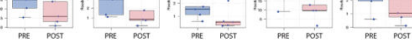

E
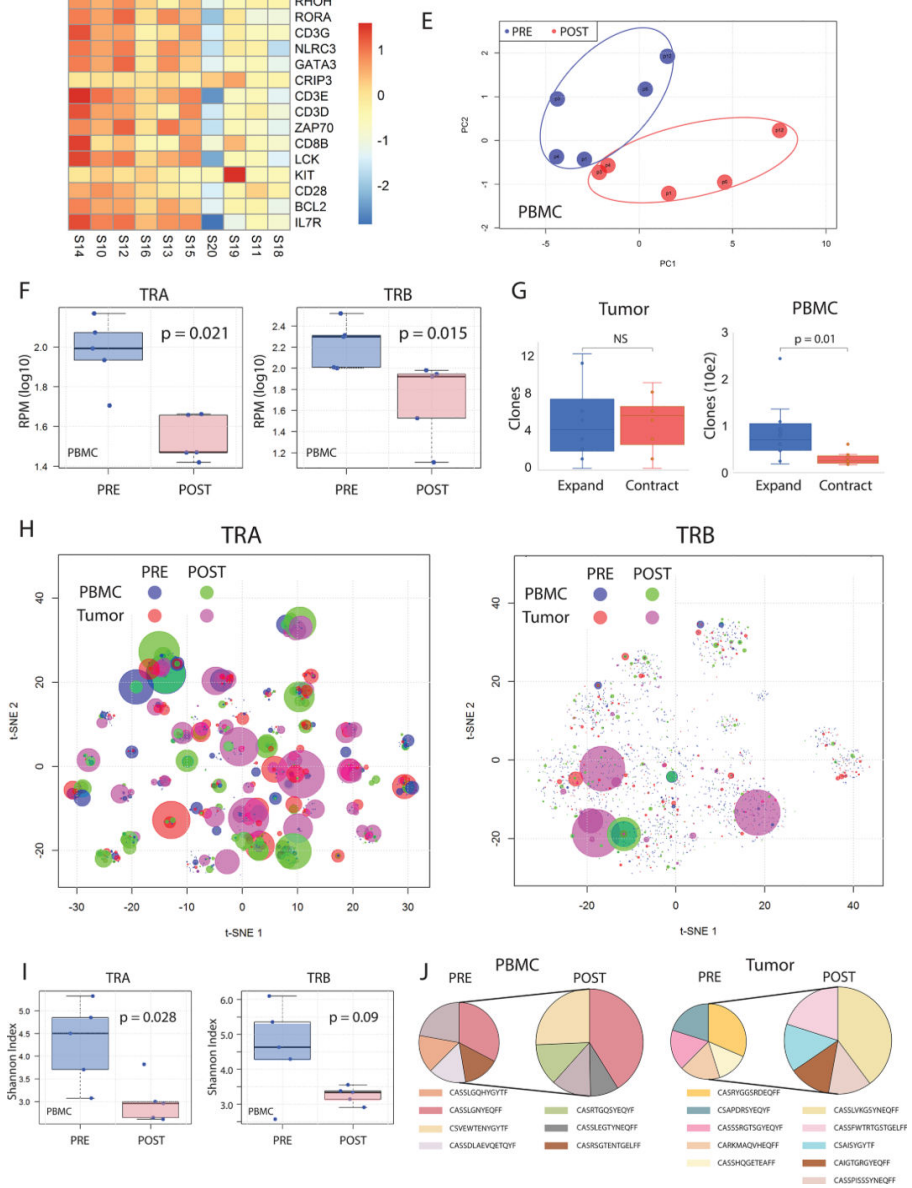

Figure 5.

Immunotherapy-induced alterations of the T cell repertoire. RNA-Seq was performed on PBMCs before and after initiation of immunotherapy. Volcano plot of resulting gene expression data reveals numerous differentially expressed genes (outlined in red) post therapy (A). Calculated Euclidean distances between T cell activaton genes (GO Biological Processes) were used to perform complete linkage clustering centered on log2-transformed data. The resulting gene expression heat map highlights gene expression differences in T cell activation genes (B). KEGG pathway analysis reveals significant alterations in immune

Clin Cancer Res. Author manuscript; available in PMC 2021 November 01. 
pathways following initiation of therapy, including the $\mathrm{T}$ cell receptor signaling pathway (C). Expression of T cell activation genes (Go Biological Processes) are presented as boxand-whisker plots where the upper and lower bars connected to each box indicate the boundaries of the normal distribution and the box edges mark the first and third quartile boundaries within each distribution (D). The dark horizontal line represents the median. Paired analysis p values were calculated using DESeq2. Principal component analysis of antigen receptor-mediated signaling gene expression data completely separates pre and post tratment samples $(\mathbf{E})$. TCR reads per million $(\log 10)$ reveals a decline in T cells after treatment $(\mathbf{F})$. TCRminer was used to extract TCR reads from RNA-Seq data and $\mathrm{p}$ values were calculated using paired Student's t-test. MiXCR was used to identify unique complementarity-determining region 3 (CDR3) sequences from RNA-Seq data and the number of expanded and contracted clones per sample were graphed for pre and post treatment samples $(\mathbf{G})$. To visualize how similar $\mathrm{T}$ cell clones were to one another with respect to their CDR3 sequences, the number of individual clones present in a particular sample, the copy number of each clone, the expansion and contractions following therapy, and the overlap between the $\mathrm{T}$ cell repertoire of the peripheral blood and the tumorinfiltrating cells, T cell repertoire galaxy plots were constructed using a modifed dimensionality reduction strategy (t-SNE) $(\mathbf{H})$. Separate plots were constructed for T cell receptor alpha and and $\mathrm{T}$ cell receptor beta (TRB) chains. In these plots the size of the circle represents the CDR3 copy number. The location of the circle represents the CDR3 sequence. Specifically, circles that are located far from one another have dissimilar CDR3 sequences whereas circles that are located close to one another have similar CDR3 sequences. Circles that share a center point have identical CDR3 sequences. Finally, the color of the circle or datapoint represents the sample (blue- PBMC pre-treatment; green- PBMC post-treatment; red- tumor pre-treatment; purple- tumor post-treatment). The Shannon diversity index was calculated for the peripheral blood $\mathrm{T}$ cell repertoire pre and post-treatment (I). As another way to visualize the alterations in the $\mathrm{T}$ cell repertoire before and after treatment, pie charts were constructed $(\mathbf{J})$. Different colors represent unique CDR3 beta sequences. The size of each colored wedge represents the copy number for that particular CDR3 sequence. 